\title{
Singular perturbation theory
}

\author{
Marc R. Roussel
}

June 21, 2004

\section{Introduction}

When we apply the steady-state approximation (SSA) in chemical kinetics, we typically argue that some of the intermediates are highly reactive, so that they are removed as fast as they are made. We then set the corresponding rates of change to zero. What we are saying is not that these rates are identically zero, of course, but that they are much smaller than the other rates of reaction.

The steady-state approximation is often surprisingly accurate, but the classical arguments lack rigor and don't lead to any satisfying mathematical generalization which can be used to improve on the approximation. There are two mathematical theories which build on the SSA and provide the required generalization:

1. Slow manifold theory: The curves or surfaces which arise from the SSA can in fact be understood as approximations to slow invariant manifolds of the differential equations. We have touched on the relevant ideas in the previous lecture.

2. Singular pertubation theory: Perturbation methods generally try to write solutions of equations as power series in a small parameter. To understand the SSA, we will find that we are led to problems in which the small parameter multiplies a derivative. These problems have an interesting structure, which is both practically and theoretically important, and to which we turn our attention in this lecture.

The two approaches are not unrelated, as we shall see in section 6.

\section{Scaling and balancing}

One of the problems with the classical SSA arguments is that they lead us to a quandary regarding the establishment of quantitative criteria for their validity. Consider the Michaelis-Menten mechanism:

$$
\mathrm{E}+\mathrm{S} \underset{k_{-1}}{\stackrel{k_{1}}{\rightleftharpoons}} \mathrm{C} \stackrel{k_{-2}}{\longrightarrow} \mathrm{E}+\mathrm{P}
$$

In this mechanism, $\mathrm{E}$ is the enzyme, $\mathrm{S}$ the substrate, $\mathrm{P}$ the product, and $\mathrm{C}$ the intermediate (enzymesubstrate/product) complex. 
The classical argument runs something like this: ${ }^{1}$ Enzymes are highly effective catalysts. We therefore expect the degradation of $\mathrm{C}$ to enzyme and product to be a fast process. Fast compared to what? Compared to formation of the enzyme-substrate complex, runs the usual answer. This argument is fine, as far as it goes, but what do we mean when we say that one chemical process is faster than another? What numbers are we comparing? It can't be rates, because in the steady state, the rates of formation and of degradation are approximately the same. It can't be rate constants, because $k_{1}$ has different units from $k_{-1}$ and $k_{-2}$.

There is no simple way out of this difficulty until we realize that we're going to get in trouble as long as we are trying to work with values which have units. If we could get rid of the units, we might be able to find things to compare such that we can make rigorous statements about what it means for one process to be fast compared to another.

Let's start by writing down the rate equations for this mechanism:

$$
\begin{aligned}
\frac{d E}{d t} & =-k_{1} E S+k_{-1} C+k_{-2} C, \\
\frac{d S}{d t} & =-k_{1} E S+k_{-1} C, \\
\frac{d C}{d t} & =k_{1} E S-k_{-1} C-k_{-2} C, \\
\frac{d P}{d t} & =k_{-2} C .
\end{aligned}
$$

Mass conservation gives us the two equations

$$
\begin{aligned}
E_{0} & =E+C, \\
S_{0} & =S+C+P .
\end{aligned}
$$

Note that these conservation relations are consequences of the differential equations since $\frac{d E}{d t}+$ $\frac{d C}{d t}=\frac{d S}{d t}+\frac{d C}{d t}+\frac{d P}{d t}=0$. Using these algebraic equations therefore doesn't change anything about the solutions. It only reduces the set of differential equations 1 to the exactly equivalent planar system

$$
\begin{aligned}
\frac{d S}{d t} & =-k_{1} S\left(E_{0}-C\right)+k_{-1} C \\
\frac{d C}{d t} & =k_{1} S\left(E_{0}-C\right)-\left(k_{-1}+k_{-2}\right) C
\end{aligned}
$$

The insight behind the SSA is that the concentrations of highly reactive intermediates like C eventually reach a rough balance between production and destruction, such that the net rate of change of $C$ is small. We want to make that balance evident. To do this, we first have to choose new measurement scales for each of the variables $(S, C$ and $t)$ such that each is of unit magnitude. In other words, we want to define new variables $s, c$ and $\tau$ by

$$
\begin{aligned}
s & =S / \tilde{S}, \\
c & =C / \tilde{C}, \\
\text { and } \tau & =t / \tilde{t},
\end{aligned}
$$

\footnotetext{
${ }^{1}$ The classical argument can be put a little better than this, but the underlying problem remains.
} 
where the tilde indicates a measurement scale whose units are the same as those of the corresponding variable and such that the new variables are, insofar as this is possible, values between 0 and 1.

It's easy to choose $\tilde{S}$ : In most experiments, we will start with $S(0)=S_{0}$, so

$$
\tilde{S}=S_{0}
$$

should be a good choice.

Choosing $\tilde{C}$ is trickier, but still not all that difficult. If we start at the initial point $(S, C)=\left(S_{0}, 0\right)$, which again is what we typically do in experiments, then $C$ will initially rise to some maximum, and then fall toward zero (the equilibrium concentration). From calculus, we know that the function $C(t)$ will reach a maximum when $d C / d t=0$, i.e. when

$$
C=\frac{k_{1} E_{0} S}{k_{1} S+k_{-1}+k_{-2}} .
$$

Note that this is the classical SSA. If we knew the value of $S$ when $C$ reaches its maximum, we could plug it in and use that as $\tilde{C}$. However, we have no easy way of estimating this value of $S$. That being said, we don't need to know the maximum value of $C$ exactly. We really only need an order-of-magnitude estimate to be used as a scale factor. We expect the maximum in $C$ to be reached early in the reaction, before much $S$ has been consumed. We therefore substitute $S=S_{0}$ into equation 5 , and get

$$
\tilde{C}=\frac{k_{1} E_{0} S_{0}}{k_{1} S_{0}+k_{-1}+k_{-2}}=\frac{E_{0} S_{0}}{S_{0}+K_{S}}
$$

where $K_{S}=\left(k_{-1}+k_{-2}\right) / k_{1}$ is the Michaelis constant, well known from classical enzyme kinetics.

It's not at all clear what we should use for $\tilde{t}$, so for now we'll just substitute equations 3 into the rate equations 2 , using the scaling factors 4 and 6 , and see what we get.

$$
\begin{aligned}
S & =s S_{0} . \\
C & =c \tilde{C}=c \frac{E_{0} S_{0}}{S_{0}+K_{S}} . \\
t & =\tau \tilde{t} . \\
\therefore \frac{d S}{d t} & =\frac{d\left(s S_{0}\right)}{d(\tau \tilde{t})}=\frac{S_{0}}{\tilde{t}} \frac{d s}{d \tau} \\
& =-k_{1} s S_{0}\left(E_{0}-c \frac{E_{0} S_{0}}{S_{0}+K_{S}}\right)+k_{-1} c \frac{E_{0} S_{0}}{S_{0}+K_{S}+k_{-2}} . \\
\therefore \frac{d s}{d \tau} & =\tilde{t}\left\{-k_{1} E_{0} s\left(1-c \frac{S_{0}}{S_{0}+K_{S}}\right)+k_{-1} c \frac{E_{0}}{S_{0}+K_{S}}\right\} .
\end{aligned}
$$

Similarly, for $C$ :

$$
\frac{d c}{d \tau}=\tilde{t} k_{1}\left(S_{0}+K_{S}\right)\left\{s\left(1-c \frac{S_{0}}{S_{0}+K_{S}}\right)-c \frac{K_{S}}{S_{0}+K_{S}}\right\}
$$

Let

$$
\alpha=\frac{S_{0}}{S_{0}+K_{S}}
$$


This is a parameter between 0 and 1 . Our two rate equations become

$$
\begin{aligned}
& \frac{d s}{d \tau}=\tilde{t}\left\{-k_{1} E_{0} s(1-\alpha c)+k_{-1} c \frac{E_{0}}{S_{0}+K_{S}}\right\}, \\
& \frac{d c}{d \tau}=\tilde{t} k_{1}\left(S_{0}+K_{S}\right)\{s(1-\alpha c)-c(1-\alpha)\} .
\end{aligned}
$$

The terms in the brace of the second of these equations are now clearly balanced: Each is made up of a product of quantities which are of unit magnitude. There only remains to choose $\tilde{t}$.

For many enzymes, the back reaction $\mathrm{C} \rightarrow \mathrm{E}+\mathrm{S}$ isn't particularly significant. Under these conditions, $d s / d \tau$ should be dominated by the first term, $-k_{1} E_{0} s(1-\alpha c) \tilde{t}$. This suggests that we should take

$$
\tilde{t}^{-1}=k_{1} E_{0},
$$

in order to bring out the balance between the derivative and this term. To put it another way, we expect $\left(k_{1} E_{0}\right)^{-1}$ to represent a slow time scale controlling the rate at which the substrate is used up. Obviously, if the above hypothesis is false for some particular enzyme, we should make a different choice for $\tilde{t}$. In any event, our choice leads to

$$
\begin{aligned}
& \frac{d s}{d \tau}=-s(1-\alpha c)+c \frac{k_{-1}}{k_{-1}+k_{-2}}(1-\alpha), \\
& \frac{d c}{d \tau}=\frac{S_{0}+K_{S}}{E_{0}}\{s(1-\alpha c)-c(1-\alpha)\} .
\end{aligned}
$$

Define

$$
\begin{aligned}
\beta & =\frac{k_{-1}}{k_{-1}+k_{-2}}, \\
\text { and } \mu & =\frac{E_{0}}{S_{0}+K_{S}} .
\end{aligned}
$$

Then we have

$$
\begin{aligned}
\frac{d s}{d \tau} & =-s(1-\alpha c)+\beta c(1-\alpha), \\
\text { and } \mu \frac{d c}{d \tau} & =s(1-\alpha c)-c(1-\alpha) .
\end{aligned}
$$

In classical enzymology, it is often said that the SSA is valid when the enzyme concentration is small. This corresponds to a small value of $\mu$. The above scaling provides a justification for this statement, as well as a precise condition for its validity: If $\mu$ is very small, then the differential equation $7 \mathrm{~b}$ is "close" to the algebraic equation

$$
0 \approx s(1-\alpha c)-c(1-\alpha)
$$

i.e. the SSA. A small value of $\mu$ implies that

$$
E_{0} \ll S_{0}+K_{S},
$$


i.e. the SSA will be valid under this condition. Note that this is a sufficient, but not a necessary condition. If we scaled time differently, we might find other conditions which lead to the validity of the SSA.

Note that there's something funny about what we're doing here. We started out with two differential equations, and ended up saying that one of the differential equations degenerates to an algebraic equation. A system of two differential equations is quite different from a single differential equation with an algebraic constraint: The first requires the values of both $s$ and $c$ to specify the initial condition, while the latter requires only a value for $s$, the value of $c$ being computed from equation 8 . We have changed the nature of the problem by considering the limit $\mu \rightarrow 0$, a limit which can never be realized in practice. Problems like this are called singular perturbation problems. Because the problem we started with and the problem we obtain in the limit in which our small parameter reaches zero are different in nature, singular perturbation problems can be very tricky. However, they show up all over the sciences, so it's well worth thinking about them a bit more.

Despite the oddity of singular perturbation problems, the use of the limit $\mu=0$ to approximate the slow manifold (which is what we get from equation 8 if we solve for $c$ ) is supported by a theorem: Consider a system of differential equations of the form

$$
\begin{aligned}
\frac{d \mathbf{x}}{d t} & =\mathbf{f}(\mathbf{x}, \mathbf{z}, t), \\
\mu \frac{d \mathbf{z}}{d t} & =\mathbf{g}(\mathbf{x}, \mathbf{z}, t) .
\end{aligned}
$$

In the singular limit $\mu=0$, we obtain the system

$$
\begin{aligned}
\frac{d \mathbf{x}}{d t} & =\mathbf{f}(\mathbf{x}, \mathbf{z}, t), \\
\mathbf{z} & =\phi(\mathbf{x}, t),
\end{aligned}
$$

where the second of these equations is the solution of $\mathbf{g}(\mathbf{x}, \mathbf{z}, t)=\mathbf{0}$. This is called the degenerate system. Finally, we define the adjoined system, which is just

$$
\frac{d \mathbf{z}}{d t}=\mathbf{g}(\mathbf{x}, \mathbf{z}, t)
$$

where now we treat $\mathbf{x}$ and $t$ as constants. ${ }^{2}$

Theorem 1 (Tikhonov's theorem) When $\mu \rightarrow 0$, the solution of the system 9 tends to the solution of the degenerate system if $\mathbf{z}=\phi(\mathbf{x}, t)$ is a stable root of the adjoined system.

(The condition regarding the stability of the adjoined system is rarely checked.) The upshot of Tikhonov's theorem is that for sufficiently small $\mu$, there is always a slow manifold whose formula is approximately given by the SSA. Further details can be obtained from Klonowski's excellent review paper [1].

\footnotetext{
${ }^{2}$ If you're wondering where the $\mu$ went in the adjoined system, the time variable can be rescaled in such a way as to make this coefficient disappear. Since stability doesn't depend on how we scale time, there's no point carrying this parameter around.
} 
Now imagine that we start an experiment at the point $(s, c)=(1,0)$ for a system with a small value of $\mu$. Initially, because $c=0$, we will have $\dot{c} \equiv d c / d \tau=1 / \mu$. This is a large (because $\mu$ is small), positive quantity. The intermediate complex concentration will therefore increase rapidly at first, with a characteristic rise time of $\mu$. However, as $c$ increases, $\dot{c}$ will become smaller. Because $\mu$ is small, the right-hand side of equation $7 \mathrm{~b}$ should also remain small, i.e. equation 8 will become valid. In other words, we can separate the time course of the reaction into two parts:

1. The rapid rise in the intermediate concentration: In chemical kinetics, we call this the induction period, or sometimes the transient. In singular perturbation theory, this is called the inner solution. ${ }^{3}$

2. A slower decay toward equilibrium during which the SSA is at least approximately valid. This is the outer solution. ${ }^{3}$

\section{The outer solution}

In this section, we obtain the outer solution to lowest order in $\mu$. This uses elementary techniques which you should have seen in your undergraduate courses. Higher order approximations can be obtained. See Heineken, Tsuchiya and Aris [2] for details.

Solving equation 8 for $c$, we get

$$
c=\frac{s}{\alpha s+1-\alpha} .
$$

Substituting this equation into the differential equation $7 \mathrm{a}$, we get

$$
\begin{aligned}
\frac{d s}{d \tau} & =-s\left(1-\alpha \frac{s}{\alpha s+1-\alpha}\right)+\beta(1-\alpha) \frac{s}{\alpha s+1-\alpha} \\
& =-\frac{s(1-\alpha)(1-\beta)}{\alpha s+1-\alpha} .
\end{aligned}
$$

This equation is easily solved by separation of variables:

$$
\begin{aligned}
\int_{s_{0}}^{s} d s \frac{\alpha s+1-\alpha}{s} & =-(1-\alpha)(1-\beta) \int_{\tau_{0}}^{\tau} d \tau . \\
\therefore-(1-\alpha)(1-\beta)\left(\tau-\tau_{0}\right) & =\int_{s_{0}}^{s} d s\left(\alpha+\frac{1-\alpha}{s}\right) \\
& =\alpha\left(s-s_{0}\right)+(1-\alpha) \ln \left(s / s_{0}\right) .
\end{aligned}
$$

The constants $s_{0}$ and $t_{0}$ represent the state of the system at some time after it has reached the manifold. These are therefore arbitrary quantities not related (at least not in any simple way) to

\footnotetext{
${ }^{3}$ The terms "inner solution" and "outer solution" are such for historical reasons: Singular perturbation problems were first considered in fluid dynamics, where the inner solution referred to the behavior near a wall, while the outer solution referred to the behavior away from the wall.
} 
the true initial conditions of the system. We can therefore combine these constants into a single, arbitrary constant $A$ :

$$
-(1-\alpha)(1-\beta) \tau+A=\alpha s+(1-\alpha) \ln s .
$$

We will determine the constant $A$ later.

\section{The inner solution}

The inner solution is a bit trickier to obtain. The problem is that the equations 7 are scaled wrong. Given our scaling of time, the rise time for the intermediate complex is $\mu$, which is a small quantity. In order to study the inner solution, we have to rescale the equations such that the rise time is not small. The easiest way to do this is as follows:

$$
\tau=\mu \theta
$$

The new time variable $\theta$ is stretched by a factor of $1 / \mu$ relative to $\tau$. Our rate equations become

$$
\begin{aligned}
\frac{d s}{d \theta} & =\mu[-s(1-\alpha c)+\beta c(1-\alpha)], \\
\text { and } \frac{d c}{d \theta} & =s(1-\alpha c)-c(1-\alpha) .
\end{aligned}
$$

Setting $\mu=0$, i.e. to the same level of approximation as our outer solution, we get

$$
\frac{d s}{d \theta}=0
$$

with equation $12 \mathrm{~b}$ remaining as is. It follows that we can, to the lowest level of approximation, assume that $s$ is approximately constant during the induction period. To distinguish this solution from the outer solution, we define $s_{\text {in }}$ to be the inner solution. Since $s=S / S_{0}$, and $S_{0}$ is the initial concentration of $\mathrm{S}, s_{\text {in }}=1$ to lowest order in $\mu$.

With $s=s_{\text {in }}=1$, equation $12 \mathrm{~b}$ reduces to

$$
\frac{d c}{d \theta}=1-c
$$

This is a simple linear differential equation which can be integrated from the initial condition $c=0$ :

$$
\begin{aligned}
\int_{0}^{c} \frac{d c}{1-c} & =\int_{0}^{\theta} d \theta . \\
\therefore \theta & =-\ln (1-c), \\
\text { or } \quad c & =1-e^{-\theta} .
\end{aligned}
$$

\section{Matching the inner and outer solutions}

Since they just represent two pieces of the same trajectory, the inner and outer solutions have to match up. This will provide us with conditions from which we will determine the parameter $A$. 
The outer solution is supposed to be valid for values of $\tau$ which aren't too small, while the inner solution is valid when $\tau$ isn't too large. To match the two solutions, we consider small values of $\tau$ and large values of $\theta$. At small values of $\tau$, the outer solution should represent early moments after the decay of the transient, while large values of $\theta$ should correspond to the very late stages of the induction period. If we have done everything right, we should be able to match up the two solutions in this intermediate regime. If we let $\tau \rightarrow 0$ and $s \rightarrow s_{\text {in }}$ in the outer solution 11 , we get

$$
A=\alpha s_{\text {in }}+(1-\alpha) \ln s_{\text {in }}=\alpha .
$$

Thus, $s$ satisfies

$$
-(1-\alpha)(1-\beta) \tau+\alpha=\alpha s+(1-\alpha) \ln s
$$

in the outer solution region.

Matching the values of $c$ yields no extra information in this case: If we put $s=s_{\text {in }}=1$ in equation 10 , we get $c=1$. If we take the limit $\tau \rightarrow \infty$ in equation 13 , we get $c \rightarrow 1$. The $c$ components of the solution therefore match up automatically.

The overall procedure for doing singular perturbation work is always as shown here, at least in outline:

1. Develop an appropriate scaling for the equations. Identify the small parameter(s).

2. Find the outer solution.

3. Find the inner solution.

4. Match up the two solutions in an intermediate time range.

The only wrinkles are that we may want the solutions developed to higher order than we have done here, and that the matching procedure is sometimes difficult to carry out properly.

\section{Geometric singular perturbation theory and the outer solu- tion}

Our work in the previous few sections focused on the temporal evolution of the system. Equation 14 for instance relates the time $\tau$ to $s$ in the outer solution. However, we have hinted that these considerations are connected to the slow manifold, a structure which is best understood in phase space. We now make this connection explicit by working out a singular perturbation expansion of the slow manifold. The theoretical framework for doing this was first provided by Fenichel [3] and is known as geometric singular perturbation theory.

Note that equation 10 suggests that the manifold can be written in the form $c=c(s)$. The manifold equation for our system would therefore be

$$
\frac{d c}{d \tau}=\frac{d c}{d s} \frac{d s}{d \tau}
$$


We have $d c / d \tau$ and $d s / d \tau$ from equations 7 . We know that, for small $\mu$, the manifold reduces to the form 10. One simple way to use this knowledge is to write the manifold as a power series in $\mu$ :

$$
c(s)=\sum_{i=0}^{\infty} \gamma_{i}(s) \mu^{i} .
$$

We should find (and will confirm) that $\gamma_{0}$ is given by equation 10 .

In order to find the unknown functions $\gamma_{i}(s)$ (written simply as $\gamma_{i}$ from here on), we substitute the power series 15 into the manifold equation. I will carry out this procedure in steps:

$$
\begin{aligned}
\frac{d c}{d s} & =\sum_{i=0}^{\infty} \frac{d \gamma_{i}}{d s} \mu^{i} . \\
\frac{d c}{d \tau} & =\frac{1}{\mu}\left\{s\left(1-\alpha \sum_{i=0}^{\infty} \gamma_{i} \mu^{i}\right)-(1-\alpha) \sum_{i=0}^{\infty} \gamma_{i} \mu^{i}\right\} \\
& =\frac{s}{\mu}-\alpha s \sum_{i=0}^{\infty} \gamma_{i} \mu^{i-1}-(1-\alpha) \sum_{i=0}^{\infty} \gamma_{i} \mu^{i-1} \\
& =\frac{s}{\mu}-(\alpha s+1-\alpha) \sum_{i=0}^{\infty} \gamma_{i} \mu^{i-1} . \\
\frac{d s}{d \tau} & =-s\left(1-\alpha \sum_{i=0}^{\infty} \gamma_{i} \mu^{i}\right)+\beta(1-\alpha) \sum_{i=0}^{\infty} \gamma_{i} \mu^{i} \\
& =-s+[\alpha s+\beta(1-\alpha)] \sum_{i=0}^{\infty} \gamma_{i} \mu^{i} . \\
\therefore \frac{s}{\mu}-(\alpha s+1-\alpha) & \sum_{i=0}^{\infty} \gamma_{i} \mu^{i-1}=\sum_{i=0}^{\infty} \frac{d \gamma_{i}}{d s} \mu^{i}\left\{-s+[\alpha s+\beta(1-\alpha)] \sum_{i=0}^{\infty} \gamma_{i} \mu^{i}\right\} .
\end{aligned}
$$

We now want to collect terms in powers of $\mu$. We could just read these terms directly from the equation. For instance, the leading terms are the $\mu^{-1}$ terms:

$$
\frac{1}{\mu}\left\{s-(\alpha s+1-\alpha) \gamma_{0}\right\}=0 .
$$

Since this equation must be true for any $\mu \neq 0$, the term in braces must be zero, i.e.

$$
\gamma_{0}=\frac{s}{\alpha s+1-\alpha}
$$

which is exactly what we said this term should be. It's not too difficult to do this provided we're only interested in small powers. However, if we want to develop a general equation which would allow us to obtain the series expansion of the manifold to arbitrary order, it is convenient to rewrite equation 16 so that each side appears as a power series in $\mu$, rather than as a complicated expression involving, among other things, a product of series. Furthermore, it is convenient to write these series so that they are sums involving $\mu^{i}$ rather than, as on the left-hand side, $\mu^{i-1}$ or other such expressions. There are rules for rewriting sums which you may have learned in some of your 
mathematics courses. If not, the following examples will hopefully let you see the logic behind these rules, which isn't too hard once you've done a few examples.

The first sum on the left-hand side of equation 16 is fairly easy to rewrite in the desired form:

$$
\sum_{i=0}^{\infty} \gamma_{i} \mu^{i-1}=\sum_{i=-1}^{\infty} \gamma_{i+1} \mu^{i}
$$

If you can't see this immediately, try writing out the first few terms of each side of this identity.

The trickiest bit is the product of sums on the right-hand side of equation 16. Here is the transformation:

$$
\left(\sum_{j=0}^{\infty} \frac{d \gamma_{j}}{d s} \mu^{j}\right)\left(\sum_{k=0}^{\infty} \gamma_{k} \mu^{k}\right)=\sum_{i=0}^{\infty} \mu^{i} \sum_{j=0}^{i} \frac{d \gamma_{j}}{d s} \gamma_{i-j}
$$

The renaming of the summation indicies on the left-hand side is a convenience introduced for clarity. The key observation is that multiplying the two sums produces terms in $\mu^{i}$ when $j+k=i$.

We're now ready to go back to equation 16 :

$$
\frac{s}{\mu}-(\alpha s+1-\alpha) \sum_{i=-1}^{\infty} \gamma_{i+1} \mu^{i}=-s \sum_{i=0}^{\infty} \frac{d \gamma_{i}}{d s} \mu^{i}+[\alpha s+\beta(1-\alpha)] \sum_{i=0}^{\infty} \mu^{i} \sum_{j=0}^{i} \frac{d \gamma_{j}}{d s} \gamma_{i-j}
$$

Reading off terms in each power of $\mu$ is now easy:

$$
\begin{array}{ll}
\mu^{-1}: & s-\gamma_{0}(\alpha s+1-\alpha)=0 . \\
\mu^{i}, i \geq 0: & \gamma_{i+1}(\alpha s+1-\alpha)=-s \frac{d \gamma_{i}}{d s}+[\alpha s+\beta(1-\alpha)] \sum_{j=0}^{i} \frac{d \gamma_{j}}{d s} \gamma_{i-j} .
\end{array}
$$

We have seen the first of these equations, and even written down its solution (equation 17). The second equation can be rewritten in the form

$$
\gamma_{i+1}=\frac{1}{\alpha s+1-\alpha}\left\{-s \frac{d \gamma_{i}}{d s}+[\alpha s+\beta(1-\alpha)] \sum_{j=0}^{i} \frac{d \gamma_{j}}{d s} \gamma_{i-j}\right\}
$$

Note that we only need to know the formulas of the $\gamma$ 's up to $i$ to determine the $i+1$ 'st term in the series. For instance, $\gamma_{1}$ is calculated by

$$
\begin{aligned}
\gamma_{1} & =\frac{1}{\alpha s+1-\alpha}\left\{-s \frac{d \gamma_{0}}{d s}+[\alpha s+\beta(1-\alpha)] \frac{d \gamma_{0}}{d s} \gamma_{0}\right\} \\
& =-\frac{s(1-\alpha)^{2}(1-\beta)}{(\alpha s+1-\alpha)^{4}} .
\end{aligned}
$$

As you can imagine, these calculations get tedious pretty quickly. In the old days, we used to fill up notebooks with perturbation series, carefully simplified by hand. It was very error-prone, and we would often spend more time verifying our answers than we did doing the initial calculation. Nowadays, we just use Maple. It's much faster, but it's still a good idea to calculate a few terms by hand to check that Maple is doing the calculation correctly. 


\section{References}

[1] W. Klonowski, Biophys. Chem. 18, 73 (1983).

[2] F. Heineken, H. Tsuchiya, and R. Aris, Math. Biosci. 1, 95 (1967).

[3] N. Fenichel, J. Diff. Eqs. 31, 53 (1979). 